\title{
Electromagnetic Tagging for Electronic Music Interfaces
}

\author{
Joseph A. Paradiso, Laurel S. Pardue, Kai-Yuh Hsiao, Ari Y. Benbasat \\ Responsive Environments Group \\ MIT Media Laboratory NE18-5FL \\ 77 Massachusetts Avenue \\ Cambridge MA 02139 USA
}

Lead author contact: joep@media.mit.edu

Tel: $+1-617-253-8988$

Fax: +1-617-225-2009

Keywords: RFID, resonant tags, EAS tags, swept tagging, tag tracking, musical controller, tangible interface, magnetic tracker

\begin{abstract}
This paper describes the development of a musical interface based on electromagnetic tagging technology, where an ensemble of passively tagged objects is identified and tracked in real time when placed in the vicinity of a reader. As the system is able to identify and update the state of many (30 or more) tags simultaneously, they can be used together in any combination - e.g., several can sit on a surface at fixed distances from the reader while others can be handheld or worn by a single user or multiple performers. This interface is able to detect both free gesture (position and orientation of the objects) as well as local or tactile variables (e.g., pressure). We describe a series of controllers that exploit the musical possibilities of this architecture - the somewhat constrained Musical Trinkets, where objects were tied to simple notes and musical effects, and its successor, the Musical Navigatrics, which enabled dynamic overdubbing and control of complex musical sequences and sonic textures. We close with a description of a very simple and inexpensive actively-tagged tracking system capable of much wider range.
\end{abstract}

To appear in the Journal of New Music Research, Special issue on New Musical Interfaces, Vol. 32, No.4, December 2003 


\section{1) Introduction}

Ever since the invention of the Theremin eight decades ago (Glinsky, 2000), several noncontact, wireless, free-gesture musical instruments have been developed that exploit a variety of sensing technologies. Some, like the Theremin, are based on electric field sensing (Paradiso \& Gershenfeld, 1997); others are active optical trackers (Rich, 1991), sonars (Gehlhaar, 1991), or vision-based systems (Zacks, 1999; Camurri, 2000). The vision-based approaches yield the richest data, enabling many different objects or body parts to be independently tracked (Paradiso \& Sparacino, 1997) without requiring special hardware to be worn or embedded in the targets. Although they are improving, vision-based systems are still often slow and fragile, becoming confused by changes in lighting or dynamic optical clutter. All of these techniques likewise require a clear line of sight between the sensor system and gesture-generating portion of the body.

Although noncontact gesture sensors can indeed make very expressive musical controllers (Paradiso 1997), they are generally notoriously difficult to virtuosically master because of the lack of any haptic or tactile reference (O'Modhrain \& Chafe, 2000). The combination of free-gesture sensing with a tangible reference has the potential of producing a very expressive electronic musical interface that also encompasses some degree of tactile precision and versatility. A generic example of this kind of combination is the Digital Baton (Paradiso \& Sparacino, 1997; Marrin and Paradiso, 1997), a handheld device that sported a set of accelerometers and a precise optical tracker for motion and position measurement together with several continuous pressure sensors for deliberate finger input. Although the handheld electronics were relatively compact, this was an active device of considerable complexity that required a wired connection. Other hybrid free-tactile gesture systems have been deployed for music [e.g., Sawada, Onoe, \& Hashimoto, (1997)], but all are active circuits that either consume power or need to be cabled.

Another way to broaden the possibilities available to a free-gesture interface is to provide an array of tethered sound-controlling objects that represent a palette of tools that a musician can grab to launch sounds and manipulate to control a musical process. An analogy for this is found in the early Archery-era concerts by John Zorn (Davis, 1991; Pareles, 1983), where he'd bring a table strewn full of various noise-making objects (e.g., bird calls, bubbling straws, reeds, shakers, percussive things) onto the stage and do extended improvised (and riveting) sets by spontaneously grabbing and playing the different implements on the table whenever the appropriate whim struck. The ability to select introduces variety, and the tactile reference afforded by the objects themselves provides a physical grounding to the gesture. 
This approach can be seen in the "Augmented Groove" project (Poupyrev, 2000) by ATR and the University of Washington, where a computer vision system recognized a set of objects based on the appearance of a printed label and, when detected, inferred their positions and orientations. Each object was used to produce or modify a particular set of musical sounds and effects, which were modulated as the object was manipulated. The objects (simple vinyl LP records in this case) were passive and extremely simple, while the large variety of different targets identified by the system led to a wide range of control parameters and produced a natural collaborative environment for multiplayer interaction.

As this example exploits optical tracking, it requires a clean line of sight, and is subject to occlusion by body parts or other objects in the field of view. One of the only approaches that doesn't suffer from this problem is magnetic tracking, as relatively lowfrequency magnetic fields pass easily through the body and other non-ferrous entities. The computer graphics industry has perfected such techniques for motion capture applications (Allen, Bishop \& Welch, 2001). These trackers, however, while quite accurate and sensitive across many meters of range, require active pickups, which involve expensive hardware and often a wired connection to a base station or master beltpack. Although we will explore such active devices at the end of this article, the bulk of this discussion will focus on a musical interface based around objects embedded with passive electromagnetic tags, which are very simple transponders that are powered by magnetic, electrostatic, or RF energy provided by a reader's antenna. These tag packages tend to be extremely compact and inexpensive. By placing such a tag on each object that one uses as a controller, one attains the potential of identifying and tracking them without needing a clear line-of-sight. Going further, if these tags respond to a local physical parameter (e.g., pressure, finger position, etc.), the objects can also be made to exhibit additional degrees of tactile expression.

This paper describes two musical environments created for the tagging interface. The first, Musical Trinkets, uses a fairly simple mapping that assigns simple notes and musical events to the tag controls, while the second, Musical Navigatrics, allows the player to exert higher-level control over arpeggios and musical textures and facilitates dynamic recording and overdubbing of musical gestures. The paper concludes with the introduction of a hardware system that uses low-cost active tagging. Although these sensor nodes require batteries or cabling, this device is capable of a much larger sensing radius.

\section{2) Passive Resonant Tag Systems}

Commercial chip-based RFID (Radio Frequency Identification) systems (Finkenzeller, 2003), while capable of addressing virtually limitless ID space, generally respond too slowly for real-time musical control, especially when multiple tags are present, 
since anticollision protocols can lead to considerable delay. Simple magnetically-coupled resonant tags (i.e., containing only an electrical or mechanical resonance without a CMOS chip) don't necessarily have this problem; if each tag has a different resonant frequency, they can all be read quickly without significant mutual interference. On the other hand, the space of available ID's is limited (determined by the width or Q of the resonance and the amount of frequency sweep), but is still ample for distinguishing several dozen objects, which is adequate for an interesting musical controller. Resonant tags are inexpensive and easily fabricated by either choosing an inductor and capacitor (their resonant frequency is determined by $[2 \pi \sqrt{L C}]^{-1}$, where $\mathrm{L}$ is the inductance and $\mathrm{C}$ is the capacitance of the electrical resonator) or trimming a magnetostrictor strip (magnetically-coupled mechanical resonators such as used in Sensormatic's UltraMax (Sensormatic, 2003) anti-shoplifting system) to the appropriate length. Since the coupling of the tags with the reader's magnetic field is dependant on orientation, objects with three orthogonal tags are able to determine inclination (relative to the local field vector) independently from range (angle can also be decoupled from range by using multiple reader coils). In addition, resonances can be made to be parametric with a local mechanical parameter such as pressure (which can detune the coil or change the capacitance of a LC resonator, for example), enabling them to also function as tactile sensors (Fletcher et al., 1997), albeit consuming a little more sweep bandwidth.

Although passive resonant tags are most commonly used in Electronic Article Surveillance (EAS) applications as shoplifting tags, they have made recent, limited inroads into entertainment, showing up as interactive pieces in board games (Dames, 1998), (Longworth, 1999), in the mallets of Don Buchla's "Marimba Lumina" MIDI controller (the O, 2000), or in the puck-like controllers of Patten and Recht's Audiopad (Patten, Recht \& Ishii, 2002). These are all close-range interactions, however, with all gesture being sensed within an inch or so of an interactive plane and precise position tracked across the surface descendants of the familiar Wacom tablet (Murakami, 1995), which similarly used magnetic tags to track and identify a set of coded pens. Our research in using tags as a musical controller conversely looked at measuring the tags across a 3D volume (e.g., a 1-foot cube) and responding to other parameters, such as orientation, proximity, and local gesture (e.g., pressure).

Although our initial work explored the use of pulse-induction ringdown tag readers (Hsiao, 2000; Paradiso, Hsiao \& Benbasat, 2002) that promised considerable sensing range, their response was prohibitively slow for prompt musical interaction with multiple tags. The passive systems described in this paper were thus all based on swept-frequency designs introduced in Hsiao (2000), Paradiso, Hsiao \& Benbasat (2001), Hsiao \& Paradiso (1999), 
Paradiso \& Hsiao (1999), Paradiso, Hsiao, Strickon, Lifton \& Adler (2000) and diagrammed in Fig. 1. This circuit uses a differentially-driven inductive bridge, where a search coil (currently 12 turns of magnet wire around a 12" diameter frame, giving roughly $130 \mu \mathrm{H}$ of inductance) is balanced against a set of reference inductors. The bridge excitation is a 20-volt peak-peak sinusoid, sweeping exponentially from 40 to $400 \mathrm{kHz}$ at a $30 \mathrm{~Hz}$ repetition rate. The bridge imbalance is synchronously demodulated and low-pass filtered to attenuate noise background. A high-pass filter then enhances abrupt changes in the bridge's null caused by variations in the current drawn by the search coil when the center frequencies of proximate resonant tags are traversed. The entire sweep is monitored by a PIC 16C73 microcomputer that logs the center frequency (corresponding to tag ID), width, and integrated height (corresponding to proximity or coupling strength) of each detected peak after each sweep, then transmits them to a host PC through a serial connection. Related architectures are used to detect LC shoplifting tags in commercial systems (Lichtblau, 1985) at higher frequencies. Fig. 2 shows a picture of the tag reader board and reader coil corresponding to the circuit of Fig. 1 - the peaks visible on the scope result from the tags that are resting inside the coil.

Although our musical applications all exploit planar coil geometries, which produce sufficiently deterministic gestural response in the vicinity of the reader, we are exploring (Hsiao \& Paradiso, 1999; Paradiso \& Hsiao, 2002) cubic geometries, with coils wound on each face. By driving coils on opposing faces together, a more uniform, higher-flux field is produced inside the cube, yielding increased measurement range and higher accuracy. Tagged objects (or fingers wearing tagged rings) can be inserted into the wireframe cube, where they are then identified and precisely tracked. Although this system has application in virtual and augmented reality, we explore potential below as a musical interface, both as a tangible controller (Ishii \& Ullmer, 1997) with tagged objects or as a modulation source (e.g., like a keyboard's left-hand controller), with tagged fingers augmenting performance on another instrument.

\section{3) Musical Trinkets}

The first sound-generating application that we built from our swept-frequency tagging system was a simple demonstration in 1995 (Paradiso, 1995) that beeped at different pitches to indicate the presence and orientation of various LEGO pieces atop a gameboard. By 1998, our hardware had evolved sufficiently to develop this into Musical Trinkets, a musical environment continuously controlled by an array of 20 tags embedded in 16 objects, as shown in Fig. 3. Two (the Cube and the Eyeball) have three embedded tags 
to determine full orientation together with proximity. One (the Pez) can also detect the position of its head as it's pulled (a ferrite bead is attached to the dispenser rod, which shifts the tune of a coil wound around the Pez's body, correspondingly changing the center frequency of its magnetic resonance). The "Ghosts" (front row) are tags that have a plastic band attached so they can be worn as rings, enabling each finger to provide independent control (earlier versions of these had coils wound around the fingers, as in Fig. 4). The other objects are single-tag items designed to be handheld and moved about or sit statically atop the reader's surface.

All objects are very simple, made from nonferrous, nonconductive plastic or resin and were either cast by us or purchased as "trinkets" at a local toy store, lending a playful air to the system's appearance. Although any of these objects can be picked up and moved freely, the design of each particular object lends itself to particular applications; some are structured to be able to sit atop the reader's table, launching background sounds and textures, while others can be rolled around, dynamically adding and modifying audio and graphic effects. As portrayed in Fig. 4, the system was augmented in 1999 with interactive graphics projected onto a frosted screen placed inside the read coil. The graphics were driven directly from the tag data, hence gave visual grounding to the interaction and emphasized the position of the reader coil, from which all proximity measurements were taken (all interaction occurred within a foot of the screen). Unlike augmented reality approaches [e.g., (Poupyrev, 2000; Longworth, 1999)], that generally require the user to look at a monitor or screen situated away from the interface or to wear potentially cumbersome goggles, all attention is focussed down towards the reader coil, where the action is occurring.

An attached PC laptop uses a custom-written $\mathrm{C}++$ program running under ROGUS (Denckla \& Pelletier, 1996) to produce the accompanying graphics and map the dynamic tag data into a MIDI stream that drives a set of music synthesizers. Referencing the set of objects in Fig. 3, the Goblins launch background droning chords (with amplitude proportional to their distance from the reader) and set the key for the notes that are played by the Ghost rings, which each fire when brought close to the reader and aligned vertically (e.g., lined up with the solenoidal magnetic field) the rings work as a free-gesture keyboard, with attack velocity determined by the speed of finger motion. The Pikachu plays a sequence of textural notes, their pitch rising with proximity. The Cube launches a low, droning tone, with pitch smoothly bending as a function of orientation (sounding much like Jon Hassell's processed trumpet (Stern, 1997). The Pez introduces a choral voice, firing another sound (a synthesized brass) when pulled, with filter cutoff depending on the extent of the pull. The other objects are modifiers, which affect the active voices. The Porcupine is 
a pitch shifter (bending pitch continuously down with proximity), the Pig produces vibrato or intervoice detune, and the Eyeball introduces audio effects (each of the three tags in the eyeball controls a different parameter in a complex patch running on an attached Lexicon LXP100 audio effects processor).

Musical Trinkets has been brought to the public several times, e.g., at SIGGRAPH 2000 (Paradiso, Hsiao \& Benbasat, 2000) in New Orleans (see Fig. 5), SMAU 2000 in Milan, and the EMP Museum in Seattle for the NIME workshop at CHI2001. As Fig. 5 indicates, the interface is highly collaborative - people gather around watching each other, then grab objects to join the interaction. The reaction to this installation has generally been very positive - people enjoy sculpting sounds and graphics with our toolkit of objects, and impromptu "jam" sessions occur frequently. The mapping, although simple, isn't entirely intuitive, however, and this installation generally requires an attendant to get participants started. The causally-generated video projection aids users in acquiring the mapping, as the visuals are tightly related to the raw tag signals (hence gesture) and generated audio.

\section{4) Tag System Performance}

In order to quantify the performance of the tag reader electronics outlined in Fig. 1, results are presented in this section that demonstrate the different aspects of tag sensing. Fig. 6 shows the range sensitivity for the three different tags in the photograph, namely an LC tag formed with a $3.5 \mathrm{~cm}$ long, $0.5 \mathrm{~cm}$ diameter, $100 \mu \mathrm{H}$ inductor (a component commonly available at any Radio Shack store) in the Cube that resonates at $75 \mathrm{kHz}$, an LC tag made from a 7-cm diameter air-core coil wound around the top of a paper cup that resonates at $200 \mathrm{kHz}$, and a $4-\mathrm{cm}$ long magnetostrictor shoplifting tag that resonates at 60 $\mathrm{kHz}$. The sweep data is acquired with a granularity similar to that attained with the PIC used in the Musical Trinkets system, namely 8-bits sampled at $12.5 \mathrm{kHz}$. Sweep data was acquired every centimeter as the tag was lifted vertically above the center of the reader coil.

The raw sweep signals (with baseline subtracted) are shown in the plots at left; traces at all sampled elevations are plotted atop one another so one can visibly see the signal grow weaker as the tag moves further away. The undershoot around the peaks, caused by the second-order analog shaping filter applied to the raw reader response, has only a limited effect on tags with nearby resonances.

The strongest signal is produced by the Cup (shown at bottom), as expected since its wide diameter enables much more magnetic flux to be captured. The first seven elevation steps, within $11 \mathrm{~cm}$ of the reader, exhibit an amplitude-saturated response, as the received signal from the Cup overwhelms the reader's analog output stage. The Cube measurements 
(middle) are from one of the three orthogonal tags mounted in the Cube's block. This tag is always aligned in this test with the reader coil's axis, hence stays parallel to the local magnetic field vector to achieve maximum response. At this gain setting, the Cube signal is always linear, as is the response from the magnetostrictor (top). As the magnetostrictor's resonance is undamped, it is seen to produce secondary "beats" after the sweep passes the $60 \mathrm{kHz}$ fundamental (the sweep frequency drops from left to right in these plots; each plot represents roughly a fifth of the full exponential sweep). Since it appears at the lower end of the sweep range, only the first magnetostrictor peak is counted here, and the ripple to the right is ignored.

The plot at right in Fig. 6 illustrates more clearly how the detected tag response varies with range (r). The vertical axis shows the inverse of the sixth-root of the integrated tag signal (where the integrated signal is the sum of all samples $s_{i}$ under a tag peak, as accumulated by the embedded software running in the tag reader of Fig. 1):

$$
Z=1 / \sqrt[6]{\sum_{\text {peak }} s_{i}}
$$

As the tag reader's transmit field strength and tag response both should vary as magnetic dipoles (hence $1 / \mathrm{r}^{3}$ ), the inverse 6 -th root of the detected signal could be expected to give a linear range response. This isn't too far from what is seen here - aside from a softening closer to the reader, the measured $\mathrm{z}$ values are reasonably linear with range. A sharp deviation in slope can be seen in the response of the Cup below $11 \mathrm{~cm}$, where the reader electronics are in saturation. Note that since the integrated peak signal is used, the system still exhibits linear behavior after entering saturation (although the saturated amplitude stays constant, the peak width still varies with tag proximity), producing a useful compression of dynamic range for strongly-interacting tags.

The detection range varies as a function of the size of the tag. With this system, the Cup can be detected beyond a foot $(30 \mathrm{~cm})$ from the reader, the tags in the Cube can be seen out to $20 \mathrm{~cm}$, and the magnetostrictor can be detected to roughly $12 \mathrm{~cm}$. The error bars presented in the range plot of Fig. 6 represent the variation $(\sigma)$ of the detected tag amplitude at each point.

The top plot of Fig. 7 shows the integrated amplitude for two of the orthogonal tags in the Cube as a function of Cube rotation around the horizontal axis (the Cube was placed about $5 \mathrm{~cm}$ above the center of the reader in these tests). As each tag comes into alignment with the vertically-oriented magnetic field vector, its response is seen to maximize. If one assumes that these signals form a quadrature pair (normalized to account for the different intrinsic coupling strength of the two tags), the lower plot shows their arctangent. Although 
this curve has significant asymmetry and nonlinear saturation behavior(caused by the displacement of the orthogonal tags in the Cube and the changing flux density created by the solenoidal reader coil) and wraps onto itself every quadrant (because of the unsigned tag response), it does give a smoothly changing response to the Cube's orientation, which is a very useful control parameter.

Fig. 8 shows the response of the Pez tag to head displacement. The plot at left shows the detected sweep signals for the tag peak as the head (hence ferrite core) is pulled out in half-centimeter increments and the Pez's center frequency shifts from 210-220 kHz. In the plot at right, which shows the sweep time of these peaks (calculated both as a simple maximum and as a centroid) we see that a useful response is produced when the head is displaced between 0.5 and $2.5 \mathrm{~cm}$, allowing a good range of continuous control. Since the centroid is able to do subsample interpolation, it produces a small continuous response for an additional centimeter of diaplacement. The sampling rate here is $25 \mathrm{kHz}$, compatible with the upgraded reader electronics in the Musical Navigatrics system described below. The system is still usable when sampling at the $12.5 \mathrm{kHz}$ rate used in the previous tests - the centroid timing looks fairly similar and the simple peak timing shows more granularity at Pez extensions beyond $2 \mathrm{~cm}$.

\section{5) Musical Navigatrics}

Although the Musical Trinkets system succeeded in public trials, its musical mapping tends to be too simple and hardwired for extended or serious use. In order to enable it to be straightforward to master, the mapping was designed to stay at a very basic level. It is an intriguing interface that hints at possibilities for virtuosic performance, but despite the variety of objects available, it often stays in too simple a sonic space. The main object that hinted at deeper interaction was the 3-axis effects-generating Eyeball - when it was introduced, subtle movements would change the audio played by the other objects in very interesting and convoluted ways. This is perhaps in accordance with the findings of Hunt, Wanderly \& Paradis (2003); a complex mapping of effects wrapped into the control of one object made the experience much more interesting to play. Our next look at tagbased musical interfaces, the Musical Navigatrics (Pardue \& Paradiso, 2002; Smith, 2002), aimed to push the hardware further and, most importantly, bring the content mapping to a deeper level.

While the original tag reader worked well, it suffered from problems of frequency drift that, over time, can cause one tag to be confused for another or to disappear altogether off the edge of the sweep (these tag readers use an analog oscillator because more stable digital waveform generators are either prohibitively expensive or create too many artifacts 
when swept). The Navigatrics reader (Smith, 2002), shown in Fig. 9, alleviates this problem by using the crystal-locked microprocessor to continually count the number of oscillator cycles across the frequency sweep and dynamically adjust the sweep amplitude and bias to compensate for any drift while retaining the original sweep profile. Also, as an additional check, this tag reader will periodically activate a set of four reference tags distributed evenly across the sweep to verify the sweep shape. This reader also features increased sampling accuracy (moving to 12 bits from 8), a roughly three times more densely sampled sweep, and improved sensitivity by sampling and digitally suppressing the often nonlinear baseline signal (the previous reader used a static threshold, hence would attenuate the sensitivity of tags resonating where the baseline dipped). This reader also directly produces a MIDI data stream, allowing standard musical mapping software to be used.

As seen in Fig. 9, this reader is also able to selectively drive up to 3 coils. Although this reduces the sweep rate per coil from $30 \mathrm{~Hz}$ to $10 \mathrm{~Hz}$ because each coil must be sequentially switched into the circuit, it provides a sufficiently prompt response for the highlevel control exercised in the musical mappings. A simple, overlapping set of three planar coils (arranged like three overlapping circles in a Venn Diagram, see Fig. 9) breaks the degeneracy of the single-coil geometry used with Musical Trinkets, which could only respond to motion along $z$. The system is now able to respond differently to tag motion in all three axes, as depicted in Fig. 9. In general, the Musical Navigatrics mappings use the net coupling of a tag to all three coils to determine the strength of the particular set of effects associated with that tag and the difference between the signals detected at each coil to determine the balance between the effects. Because the magnetic flux bends to return around the coils, the derived coordinates are nonlinear and somewhat coupled - they are, however, quite smooth and deterministic, hence adequate for deliberate musical interaction.

Musical Navigatrics uses 12 objects embedded with 14 tags to control over 35 different variables, including arpeggiation, audio filters and effects, tempo adjustment, and sequencing controls with which to record, overdub, and playback any gesture. It is much less restricted than Musical Trinkets, enabling a player to assemble and manipulate sophisticated sonic structures. The mapping is all done in MAX (Cycling74, 2003) and the synthesis in Reason (Propellerheads, 2003), both running on a Macintosh G4 laptop. Fig. 10 shows the Musical Navigatrics system in action, as demonstrated at the NIME 2002 Conference in Dublin (Pardue \& Paradiso, 2002). The tags are embedded into flatbottomed, furry objects that we call "fuzzies", each of which is easily distinguished by its color. As Musical Navigatrics is capable of producing complex soundstreams, it is well suited to solo playing. A difficulty here is the fact that two hands only allow easy and dexterous grasp of two fuzzies. To alleviate this, each LC tag is equipped with a switch that 
shorts the tag circuit, hence deactivates it. This allows the player to position a fuzzy appropriately for a desired effect, then hit the switch with a thumb or finger, latching its settings and allowing another fuzzy to be grabbed for modifying the sound in a different manner.

The fuzzies fall into 3 primary categories: sequencing controls, arpeggiation controls, and effects controls. Sequencing controls allow the recording, overdubbing, and playback of stored gestures, MAX sequences, and Reason sequences. Arpeggiation fuzzies change the arpeggiation style on up to three voices, two of which are pre-loaded and one of which is determined through the tag's motion. Finally, there are six effects objects controlling eight audio effects that may affect either one selected voice at a time or the entire ensemble with various filters, delay and reverbs.

The sequence control objects consists of the play fuzzy, record fuzzy, and the tempo and rhythm fuzzy. These three objects are the only objects where expressed gestures are not stored in record mode. Despite the apparently simple nature of these functions, there is quite a bit of complexity available in playback and record styles. Play will cause any gestures that have been recorded to immediately loop back. If an already recorded tag is being used during playback, its new motion will be used rather than the recorded version. Introducing the record fuzzy triggers recording, providing introductory clicks for cueing tempo and downbeat and recording as long as the record fuzzy is present. Currently, Navigatrics records only in $4 / 4$ based meters. If the play fuzzy is also being used, the record mode changes to overdub and records anything played, allowing layering of expressive gestures. The last control tag is the tempo and rhythm fuzzy. Although play and record work essentially just as on/off switches, the tempo fuzzy is a latchable tag that exhibits continuous control. The coupling strength from the three reader coils is combined (producing a $z$-axis position estimate) to determine the tempo, while the position of the tempo fuzzy in the horizontal plane crossfades between different drum patterns (positioning the tempo fuzzy over coil 1 inhibits the drums all together).

The second set of fuzzies controls the arpeggiating voices. There are three of these, all latchable, each activating and controlling a different melodic line. The primary two arpeggiation fuzzies have three degrees of freedom, which are loosely tied to an $(x, y, z)$ coordinate mapping. These three dimensions determine the shape of the arpeggio, the number of octaves the arpeggiation reaches (up to 4), the number of notes per octave, and the direction/order of the arpeggio. The direction/order variable determines whether the arpeggio goes up or down, up and down, or whether it goes up in steps, e.g., 4 notes up, 1 down, 3 notes up, 1 down, 3 more notes up, etc. The basis or key of the scale for arppegiation is determined by single line loops that are preloaded into MAX. The third 
arpeggiation fuzzy controls only two arpeggiation variables: direction and number of notes per octave. It is fixed to a two-octave range. This frees up a third degree of freedom, height (z), which can be used to traverse a note table and compose a single line melody to arpeggiate over. This allows for easy (yet limited) melody making if there are no appropriate preloaded sequences available. Resting an arpeggiation fuzzy inside a reader coil will also change its mode of operation; A fuzzy resting atop the reader coil is considered a "selected voice" for effects purposes, as described below.

The last set of objects is the group of six effects fuzzies. These latchable tags provide a wide range of timbral expression through modulation of volume, pan, reverb, delay, chorus, flanging, a "drone", arpeggio speed, phasing, distortion, and filter controls. Through the ability to loosely place the tag in 3 dimensional space, it is possible to use one fuzzy to map 3 variables (or more if the fuzzy has multiple tags) per effect, allowing the timbre to be multimodally altered. For instance, the filter fuzzy is able to change several parameters that define the filter's response characteristic in an understandable and repeatable way; it can also be assigned to particular voices. There are primarily two categories of effects fuzzies: those that control voice effects and those that control master effects. The filter fuzzy, for example, controls a voice effect. Using a voice effect fuzzy while there are one or two arpeggiation fuzzies present will only change effects on those specific voices. If all or none of the arpeggiation fuzzies are present, then the voice effects will be heard on all voices. With the ability to select a voice in this fashion, multiple effects can be recorded on a specific arpeggiation voice and sequence. The voice effect fuzzies include volume and pan (which uses a similar mapping to the one for tempo and drums; here magnitude $(z)$ controls volume and planar position controls pan), phasing and distortion effects, and voice filtering. Master effect fuzzies are those that do not reference selected voices. Common delay, reverb, and chorus/flanging are all tied to one single fuzzy, the cube. The cube itself is constructed so that there is a different tag aligned with each axis, hence the effects change not only by translation (as with the other tags), but also by rotation, providing a very expressive interaction. The other two master effects fuzzies are standard single tag objects. The "drone" fuzzy evokes a non-arpeggiated voice. It can either be a simple single note drone or it can be a preloaded sequence. Introducing the drone fuzzy will trigger the drone sound to start; the drone is then modified by moving its fuzzy about. The arpeggiation speed fuzzy modifies the length of the arpeggiated notes from as long as a half note to a thirty-second note. This fuzzy has a very simple mapping, as the speed of each arpeggiation is determined by the corresponding coupling strength $\left(z_{\mathrm{i}}\right)$ measured at each of the three coils. 
Overall, Musical Navigatrics has achieved its goal of demonstrating a much more complex musical environment than the Musical Trinkets. Although the details can be difficult to master immediately, nearly any gesture produces an interesting response, and after working with the system for a while, the different control objects are intuitively associated with particular effects - one gets better with practice.

\section{6) Low-Cost Active Magnetic Tagging}

As discussed above, passive magnetic tags have several advantages, namely their small size, minimal complexity, and lack of a battery or local power source. The major factor restricting their applicability is their limited read range; the readers used in the projects discussed above had a maximum range of circa $20 \mathrm{~cm}$ for a $2.5 \mathrm{~cm}$ long ferrite-core tag inductor. Since the reader must both power the tag and receive its response, the signal strength generally falls with the sixth power of tag-to-reader distance. Although opposing differential drive coils, as in (Paradiso \& Hsiao, 2002), can increase the transmit range through magnetic field shaping, they can be physically cumbersome for many applications, as the action needs to take place between the coils. Commercial ringdown reader systems (Sensormatic, 2003) are able to detect magnetostrictor tags out to a few meters, but the magnetostrictors tend to be somewhat difficult to tune and, as mentioned earlier, this technique provides prohibitively slow updates for multiple tags.

One way to obtain much more range is by using an active tagging system. The design that we describe here is diagrammed in Fig. 11; it uses a set of reference transmit coils at a fixed point in the tracking space and a mobile triad of small pickup coils that receive the transmit flux and boost the induced signals with a local amplifier. The drawback here is that the pickup device requires a power source, provided either by a battery or wired connection. The advantage is that the read range is dramatically increased.

Commercial magnetic tracker systems used for motion capture in computer graphics systems (Allen, Bishop \& Welch, 2001; Murry \& Schneider, 1995) work via this principle. They are, however, often prohibitively expensive, typically costing over several thousand dollars. As such tracking capabilities and devices are frequently of interest to developers of musical interfaces (e.g., (Goudeseune, Garnet \& Johnson, 2001), where musical effects are mapped to spatial position), we briefly overview a very low-cost active magnetic tracker (costing well below $\$ 100$.) that we have developed and applied.

Our system is in two parts; the reference coil driver and tag receiver. The former is extremely simple, consisting of a 24-Volt peak-peak, push-pull, bipolar, Class A-B driver (a standard power amplifier configuration) connected to each coil. The coils are run at a fixed frequency here, namely $170 \mathrm{kHz}$, which was away from ambient interference (namely 
caused by video monitors) and within the range of the inexpensive linear electronics that we used. The coils are nonresonant, enabling them to be quickly pulsed at the drive frequency without needing a synchronously-switched Class E amplifier (Sokal \& Sokal, 1975). In our application, we drive them round robin, first transmitting from $B_{1}$ for circa $2 \mathrm{~ms}$ (followed by $4 \mathrm{~ms}$ of data transfer), then $\mathrm{B}_{2}$, then $\mathrm{B}_{3}$, giving us three successive, independent magnetic field references.

The receive electronics, the analog portion of which is diagrammed in Fig. 12 and designed to run off a low-power 5 Volt supply, are hardly more complicated. Pickup coil L1 is a simple $100 \mu \mathrm{H}$ ferrite-core inductor; when combined with $10 \mathrm{nF}$ capacitor $\mathrm{C} 1$, it forms a resonant tank circuit that responds to the $170 \mathrm{kHz}$ excitation coupled in from the reference coils while rejecting out of band background. U1A is a noninverting amplifier that provides $33 \mathrm{~dB}$ of gain for small amplitudes and $7 \mathrm{~dB}$ for large amplitudes (allowing the active tag to approach the drive coils without saturating the signals); this gain shaping is performed by LED1 working as a switch at roughly 2.5 volts in the feedback of U1A. The amplitude of the resulting signal is extracted by the peak detector formed by D3, C6, and R2 and further conditioned by the low-frequency amplifier U2A, which buffers the high-impedance peak detector, corrects for the signal's baseline, and provides a little more gain. Note that because we use a simple absolute-value detector here via D3, the system is unable to detect the phase of the received signal, hence can not determine the signed orientation (e.g., one can't infer if the device is rightside up or upside down - both states wrap to the same set of signals). Although it introduces a little more complication, the use of a synchronous detector (Paradiso \& Gershenfeld, 1997) in place of D3 would recover the signed orientation and improve the signal/noise ratio considerably. Synchronous detectors, however, require a phase reference. This is easily available from the coil driver in a wired system, but would be slightly more complicated for a wireless architecture.

A minimal microprocessor (the ADuC812 microconverter from Analog Devices) digitizes the resultant signals into 12 bits. For each reference coil pulsed, the signals produced by each pickup coil, processed by the electronics in Fig. 12, are sampled and sent to a host computer. This results in a matrix of 9 values that can be used to estimate the position and absolute orientation of the pickup tag package. We use a very simple calculation to determine the coordinates, namely the quadrature sum of the signals detected over all pickup coils corresponds to the distance from a particular drive coil (compensated for nonlinear rolloff) and the ratio of the signal from each pickup coil to the quadrature sum of all three determines positive direction cosine of the pickup tag package relative to the local magnetic field vector. This straightforward parameterization responds smoothly to gesture and gives reproducible results appropriate for many musical interactions that 
explore an activated space. As magnetic fields are dipole in nature, both the flux magnitude and direction change as a function of distance, however, hence the matrix of measurements must be processed and potentially iterated through more complex algorithms (Jones, 1988) if accurate coordinates are desired. Although special reference coil geometries e.g., collectively driven opposing coils as in Paradiso \& Hsiao (2002) or Herrera, (2001) can produce a more uniform field and reduce the need for such corrections, they tend to be impractical for many applications, as mentioned earlier. As with any AC magnetic field application, ferrous and conductive metal can perturb the field lines and distort the measurements. A calibration procedure is thus required if the system is deployed with significant amounts of such materials nearby.

The resulting tracking system was used in a design installation called Atmosphere (Atmosphere, 2001), which was shown at the Museum of Modern Art (MOMA) in Manhattan as a part of the Workspheres (Antonelli, 2001) exhibition that ran in FebruaryApril of 2001. Although Atmosphere did not produce any audio, it did control sophisticated interactive graphics. A collaboration between the Media Lab's Responsive Environments and Aesthetics \& Computation Groups with Steelcase, Inc., Atmosphere was a whimsical look at a calendar application. With a very stylized interpretation of a datebook projected on a large rear screen, users would navigate through it by moving and rotating a PDA (Personal Digital Assistant) about (as measured by the magnetic tracker), selecting particular appointments by manipulating the PDA controls. A complete Atmosphere interaction station can be seen at right in Fig. 13 - the installation included three such podiums, all facing the large projection. The triad of 1 -foot diameter tracker reference coils is obvious in this photo. Because the gooseneck limited the range of PDA displacement to a couple of feet, the reference coil drive voltage was attenuated here to only a few volts. When the drive voltage was modestly increased, however, to circa 20 volts, the circuit of Fig. 12 was able to sense the resultant magnetic flux up to at least four meters away, providing much larger interaction range than the passive tag systems described previously.

Fig. 13 (left) shows the active tag package integrated into the PDA case. The three pickup coils, oriented along different axes, are labeled. Ideally, all three coils should be very close to one other or wound along the same form (Herrera, 2001) to reduce collocation errors. The physical constraints of the PDA mount, however, necessitated that the pickup tags were distributed across the PDA case (the measurements were still adequate, however, for this application). At bottom left, one can see the circuit card that contained three channels of the analog electronics (Fig. 12) and the microprocessor. This card was fairly compact, measuring 3" 31.5 ", and a tighter board layout could reduce it easily by more than half. 
Our resultant system was able to provide full-state measurement updates at $55 \mathrm{~Hz}$ per interaction station when the tag package's microprocessor communicated with a host PC over a 19.2 kbaud serial line (of course, the tracking information can be used locally by the PDA as well - many useful applications don't need a datalink). A wired connection was used in this example, with cables hidden in the gooseneck seen holding the PDA at right in Fig. 13. As we needed to power the PDA and keep it from being stolen, a tether was mandatory, and there was nothing to be gained by going wireless. A wireless link would be fairly trivial to implement, however, using an inexpensive, compact, off-the-shelf RF modem [e.g., Eady, (1998)] or any of the many low power radio tranceiver chips and modules now commercially available, especially if signed orientation is unimportant and there is no need for a phase reference (the circuit of Fig. 12 can then be used directly). Once an RF link is installed, other sensors can easily be added as in Paradiso, Hsiao, Benbasat \& Teegarden, (2000) and their signals also encoded into the wireless data stream, allowing for other (e.g., tactile) degrees of freedom and multimodal local control. As the read circuit requires very little power, it can easily last for many hours or days on a modest battery, creating a compact and inexpensive, wireless active magnetic tag that can track across a large activated space for a variety of interactive music and media applications.

\section{7) Conclusions}

We have built systems that are able to continuously identify, track and monitor the state of many wireless magnetically-coupled tags across an activated 3D volume. Such capabilities have enabled us to construct a very new kind of electronic music interface. Taking inspiration from acoustic improvisers who perform using a table strewn with soundmaking paraphernalia [e.g., Davis (1991)], we have created analogous electronic environments that combine the ability to make a wide selection across a tangibly-manifested sonic palette with complex multimodal control capability that can be embedded in handheld objects. Although our initial Musical Trinkets installation was quite simple, audiences at several worldwide venues found it compelling both to watch in performance and interact with directly. Tangible music interfaces are intrinsically collaborative. This is perhaps especially true for this one, as any activated object will produce and sculpt sound when merely picked up and moved about - the barriers to entry are very low and the sonic rewards considerable.

As the subsequent Musical Navigatrics environment offered a much deeper musical interaction, proficiency would improve with practice. Although the possibilities for multimodal control were large, the number of effects controllable at one time is limited by the number of hands (hence players) to grab tagged objects. In Musical Navigatrics, this is 
somewhat addressed by the ability to latch control in mid gesture with the embedded switch, providing an intuitive means of successively building sophisticated timbral structures. The end result is a powerful, expressive, and easy-to-use free-gesture musical performance controller.

This work can be pushed forward along several directions. One tack that deserves much more attention is the physical design of the objects themselves. Although their whimsical nature gives the interaction a playful character, the toys appropriated for Musical Trinkets and the fuzzies used by Musical Navigatrics aren't optimized to their task; a main factor in their selection was the ease with which a tag could be embedded into them. Ideally the objects should bear a metaphoric visual and tactile resemblance to their function, be easily distinguished, and likewise be designed with ergonomics in mind, e.g., fitting properly in the hand across their modes of use, granting intuitive access to the latching switches and control axes, perhaps being able to easily grab and control several at once. Similarly, the tag reader can be redesigned to accommodate many more tags, enabling additional objects to be introduced. An authoring environment is likewise required that can easily allow a composer or musician to adapt the tag interface to their own musical vision. Although Musical Navigatrics, with its dynamic sequencing capability, is perhaps a step in that direction, one can certainly go much further.

By their nature, small passive magnetic tags, such as those used in this work, are limited to relatively small activated spaces and stop working when moved more than a meter or so from the reader in the best cases. We have thus introduced a very low cost, compact active tagging system that can respond to gesture across a much larger space, albeit with a limited amount of extra complication. Such a system will be of use in augmented-reality or spatially situated musical environments e.g., Goudeseune, Garnett \& Johnson (2001) where the expense of commercial motion capture equipment can be prohibitive.

\section{8) Acknowledgements}

As the work described here has spanned many years, we have many individuals and entities to recognize for stimulating discussions and project support. Accordingly, we thank our Responsive Environments Group colleagues, especially Mark Feldmeier, Leila Hasan, and Josh Lifton, and students from other Media Lab groups, notably Dan Stiehl for help in casting objects and embedding appropriate tags, Marc Downie for graphical content used with the Trinkets system, and Matt Hancher for building and characterizing test coils. In the Atmosphere project, we were happy to work with most of the Media Lab's Aesthetics \& Computation Group, and particularly acknowledge John Maeda, Ben Fry, and Max Van Kleek. Steelcase, especially Joe Branc and Charlie Forslund, were wonderful collaborators 
on Atmosphere, and discussions with Hap Patterson of Sensormatic have been appreciated throughout the course of our work with magnetic tags. We appreciate the support of the Things That Think Consortium and the other sponsors of the MIT Media Laboratory. Video clips showing the projects discussed in this paper can be accessed at: http://www .media.mit.edu/resenv/tags.html.

\section{9) References}

Allen, B., Bishop, D., Welch, G. (2001). Tracking: Beyond 15 Minutes of Thought. Volume 11 of SIGGRAPH 2001 Course Notes. Los Angeles: ACM SIGGRAPH Press.

Antonelli, P., ed. (2001). Workspheres: Design and Contemporary Work Styles. New York: Museum of Modern Art Publications, May-June Issue, Vol. 102, no. 3. See also: http://www.moma.org/exhibitions/2001/workspheres/index.html

Atmosphere (2001). See: http://acg.media.mit.edu/projects/atmosphere/

Camurri, A., et al. (2000). A Real-Time Platform for Interactive Dance and Music Systems. In Proc. of the 2000 International Computer Music Conference (pp. 262-265). San Francisco: International Computer Music Association.

Cycling74 (2003). See: http://www.cycling74.com

Dames, A.N. (1998), Position Encoder, U.S. Patent No. 5,815,091.

Davis, F. (1991). 'Zorn' for 'Anger.' The Atlantic Monthly, 267, No. 1 (January), pp. 97100.

Denckla, B. and Pelletier, P. (1996). The technical documentation for "Rogus McBogus," a MIDI library. Available at: http://www.media.mit.edu/hyperins/rogus/home.html

Eady, F. (1998). RF Telemetry; Part 1: Theory and Implementation. Circuit Cellar Ink, No. 90 (January), pp. 61-67.

Fletcher, R., Levitan, J., Rosenberg, J., Gershenfeld, N. (1997). Application of Smart Materials to Wireless ID Tags and Remote Sensors. In Materials for Smart Systems II (pp. 557-562). Materials Research Society: Pittsburgh, PA.

Finkenzeller, K. (2003). RFID Handbook: Fundamentals and Applications in Contactless Smart Cards and Identification. New York: John Wiley \& Sons.

Gehlhaar, R. (1991). SOUND=SPACE: an Interactive Musical Environment. Contemporary Music Review, 6(1), pp. 59-72.

Glinsky, A. (2000). Theremin: Ether Music and Espionage. Chicago: University of Illinois Press.

Goudeseune, C., Garnett, G., Johnson, T. (2001). Resonant Processing of Instrumental Sound Controller by Spatial Position. In ACM CHI 2001 Conference - Workshop on New Interfaces for Musical Expression (NIME2001), April 1-2. See: http://www.csl.sony.co.jp/person/poup/research/chi2000wshp 
Herrera, J. (2001). Magnetic Position Tracker. Circuit Cellar INK, Issue \#133, (August), pp. 22-33.

Hunt, A., Wanderly, M., Paradis, M. (2003). The Importance of Parameter Mapping in Electronic Instrument Design. Journal of New Music Research, this issue.

Hsiao, K., Paradiso, J. (1999). A New Continuous Multimodal Musical Controller Using Wireless Magnetic Tags. In Proceedings of the 1999 International Computer Music Conference (pp. 24-27), San Francisco: International Computer Music Association.

Hsiao, K. (2000). Fast Multi-Axis Tracking of Magnetically Resonant Passive Tags: Methods and Applications. MEng. Thesis, MIT Department of Electrical Engineering and MIT Media Laboratory.

Ishii, H. and Ullmer, B. (1997). Tangible Bits: Towards Seamless Interfaces between People, Bits and Atoms. In Human Factors in Computing Systems (pp. 234-241), New York: ACM Press.

Jones, H.R. (1988). Method and apparatus for determining remote object orientation and position. US Patent No. 4,737,794, 1988.

Lichtblau, G.J. (1985). Resonant Tag and Deactivator for use in Electronic Security System. US Patent No. 4,498,076.

Longworth, J. (1999). Look Ma, No Wires! Zowie Launches Next Wave of Smart Toys. Kidscreen. July 1, p. 18.

Marrin, T., Paradiso, J. (1997). The Digital Baton: a Versatile Performance Instrument. In Proc. of the 1997 International Computer Music Conference (pp. 313-316). San Francisco: International Computer Music Association.

Murakami, A., et al. (1995). Position Detector. US Patent No. 5,466,896.

Murry, H., Schneider, M. (1995). Virtual Reality Position Tracking. Circuit Cellar INK. Issue \#60 (July), pp. 24-29.

the O, Larry the (2000). Nearfield Multimedia Marimba Lumina: This is not your mother's MIDI controller. Electronic Musician, 16(6), p. 138.

O'Modhrain, M.S., Chafe, C (2000). The Performer-Instrument Interaction: A Sensory Motor Perspective. In Proc. of the 2000 International Computer Music Conference (pp. 145-148) San Francisco: International Computer Music Association.

Paradiso, J.(1995). See: http://www.media.mit.edu/resenv/Tags/Lego-demo/sld001.htm

Paradiso, J. (1997). Electronic Music Interfaces: New Ways to Play. IEEE Spectrum, 34(12), pp. 18-30.

Paradiso, J., Gershenfeld, N. (1997). Musical Applications of Electric Field Sensing. Computer Music Journal, 21(3), pp. 69-89.

Paradiso, J., Sparacino, F. (1997). Optical tracking for music and dance performance. In A. Gruen, H. Kahmen (eds.). Optical 3-D Measurement Techniques IV (pp. 11-18) Heidelberg: Herbert Wichmann Verlag. 
Paradiso, J., Hsiao, K. (1999). Swept-Frequency, Magnetically-Coupled Resonant Tags for Realtime, Continuous, Multiparameter Control. In Human Factors in Computing Systems; CHI99 Extended Abstracts (pp. 212-213), New York: ACM Press (1999).

Paradiso, J., Hsiao, K., Benbasat, A. (2000). Musical Trinkets: New Pieces to Play. In SIGGRAPH 2000 Conference Abstracts and Applications (p. 90). New York: ACM Press.

Paradiso, J.A., Hsiao, K., Benbasat, A.Y., Teegarden, Z. (2000). Design and Implementation of Expressive Footwear. IBM Systems Journal. Vol. 39, Nos. 3\&4, pp. 511-529.

Paradiso, J., Hsiao, K., Strickon, J., Lifton, J., Adler, A. (2000). Sensor Systems for Interactive Surfaces. IBM Systems Journal, Volume 39, Nos. 3 \& 4, pp. 892-914.

Paradiso, J., Hsiao, K., Benbasat, A. (2001). Tangible Music Interfaces Using Passive Magnetic Tags. In ACM CHI 2001 Conference - Workshop on New Interfaces for Musical Expression (NIME2001), April 1-2, 2001. See:

http://www.csl.sony.co.jp/person/poup/research/chi2000wshp/

Paradiso, J.A., Hsiao, K. (2002). Multiple-Axis Tracking of Passive Resonant Structures. US Patent No. 6,404,340.

Pardue, L.S., Paradiso, J.A. (2002). Musical Navigatrics: New Musical Interactions with Passive Magnetic Tags. In Proceedings of Conference on New Interface for Musical Expression, NIME '02 (pp. 168-169). See: http://www.mle.ie/nime/.

Pareles, J. (1983). Concert: Sounds of Staley and Zorn. New York Times, December 4, 1983, p. 93.

Patten, J., Recht, B., Ishii, H. (2002). Audiopad: A Tag-based Interface for Musical Performance. In Proceedings of Conference on New Interface for Musical Expression, NIME '02 (pp. 11-16). See: http://www.mle.ie/nime/

Poupyrev, I. (2000). Augmented Groove: Collaborative Jamming in Augmented Reality. In SIGGRAPH 2000 Conference Abstracts and Applications (p. 77). New York: ACM Press.

Propellerheads (2003). See: http://www.propellerheads.se

Rich, R. (1991). Buchla Lightning MIDI Controller. Electronic Musician, 7(10), pp. 102108.

Sawada, H. Onoe, N. Hashimoto, S. (1997). Sounds in Hands - a Sound Modifier using Datagloves and Twiddle Interface. In Proceedings of the 1997 International Computer Music Conference pp. 309-312. San Francisco: International Computer Music Association.

Sensormatic (2003). See http://www.sensormatic.com/EAS/EAS.asp

Smith, L.P. (2002). Development of An Improved Swept RF Tagging System and its Musical Applications. MEng Thesis, MIT Department of Electrical Engineering and MIT Media Laboratory.

Sokal, N.O. and Sokal, A.D. (1975). Class E - A New Class of High-Efficiency Tuned Single-Ended Switching Power Amplifiers. IEEE Journal of Solid-State Circuits, Vol. SC10, pp. 168-176.

Stern, T. (1997). Jon Hassell. Perfect Sound Forever. Online Magazine (July). See: http://www.furious.com/perfect/hassell.html 
Zacks, R. (1999). Dances with Machines. Technology Review, (May/June), pp. 58-62. 


\section{FIGURE CAPTIONS:}

Figure 1: Block diagram of the swept-frequency tag reader

Figure 2: Swept frequency tag reader board, coil, and scope trace showing response (labeled) to three tags

Figure 3: Ensemble of tagged objects that control Musical Trinkets

Figure 4: Musical Trinkets running with interactive graphics

Figure 5: Musical Trinkets at SIGGRAPH 2000

Figure 6: Range response for three different types of tags

Figure 7: Response of two orthogonal tags in the Cube to rotation at fixed range

Figure 8: A wireless pull sensor - response of the Pez tag to head displacement

Figure 9: Block Diagram for the Musical Navigatrics tag reader and read coil geometry

Figure 10: Playing Musical Navigatrics at NIME2002 in Dublin

Figure 11: Coil schematic for active tagging scheme. The large orthogonal reference coils $\left(B_{1}, B_{2}, B_{3}\right)$ successively induce signals into the small orthogonal reference coils $(x, y, z)$, which are actively amplified, detected, and digitized.

Figure 12: Simple active read electronics conditioning each pickup coil

Figure 13: Active magnetic tracker motoring PDA location in Atmosphere at MOMA 


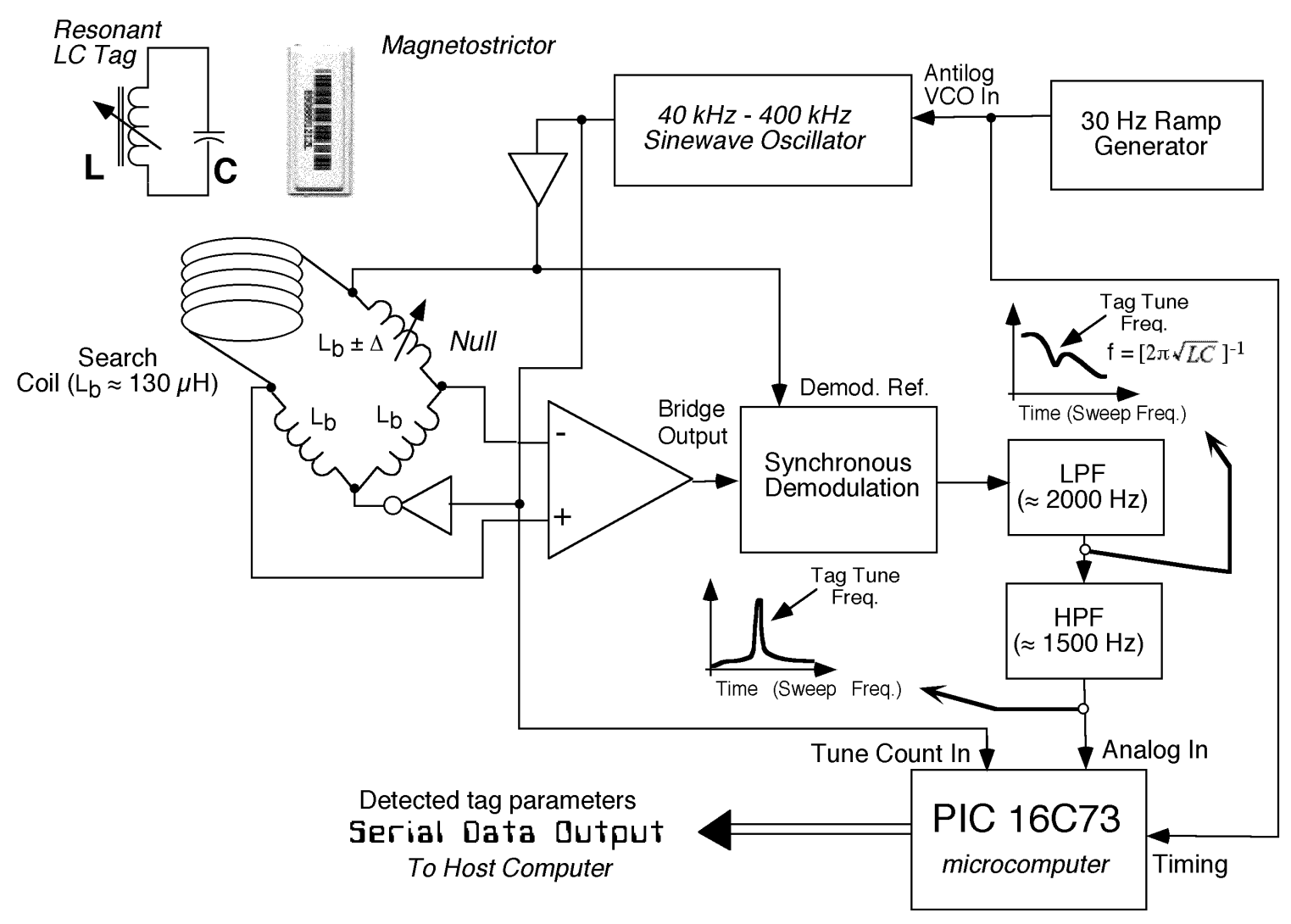

Figure 1: Block Diagram of the swept frequency tag reader 


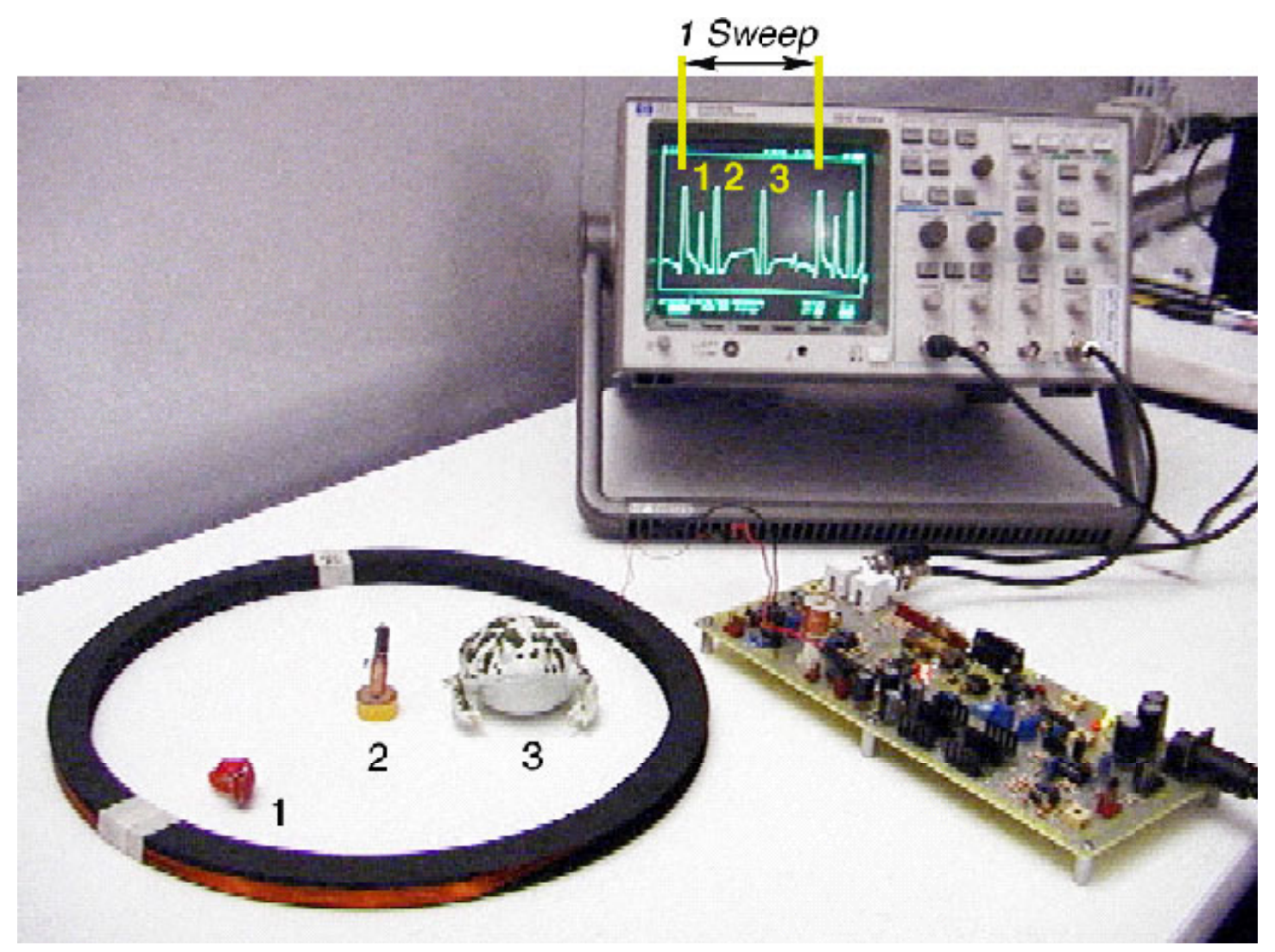

Figure 2: Swept frequency tag reader board, coil, and scope trace showing response (labeled) to three tags

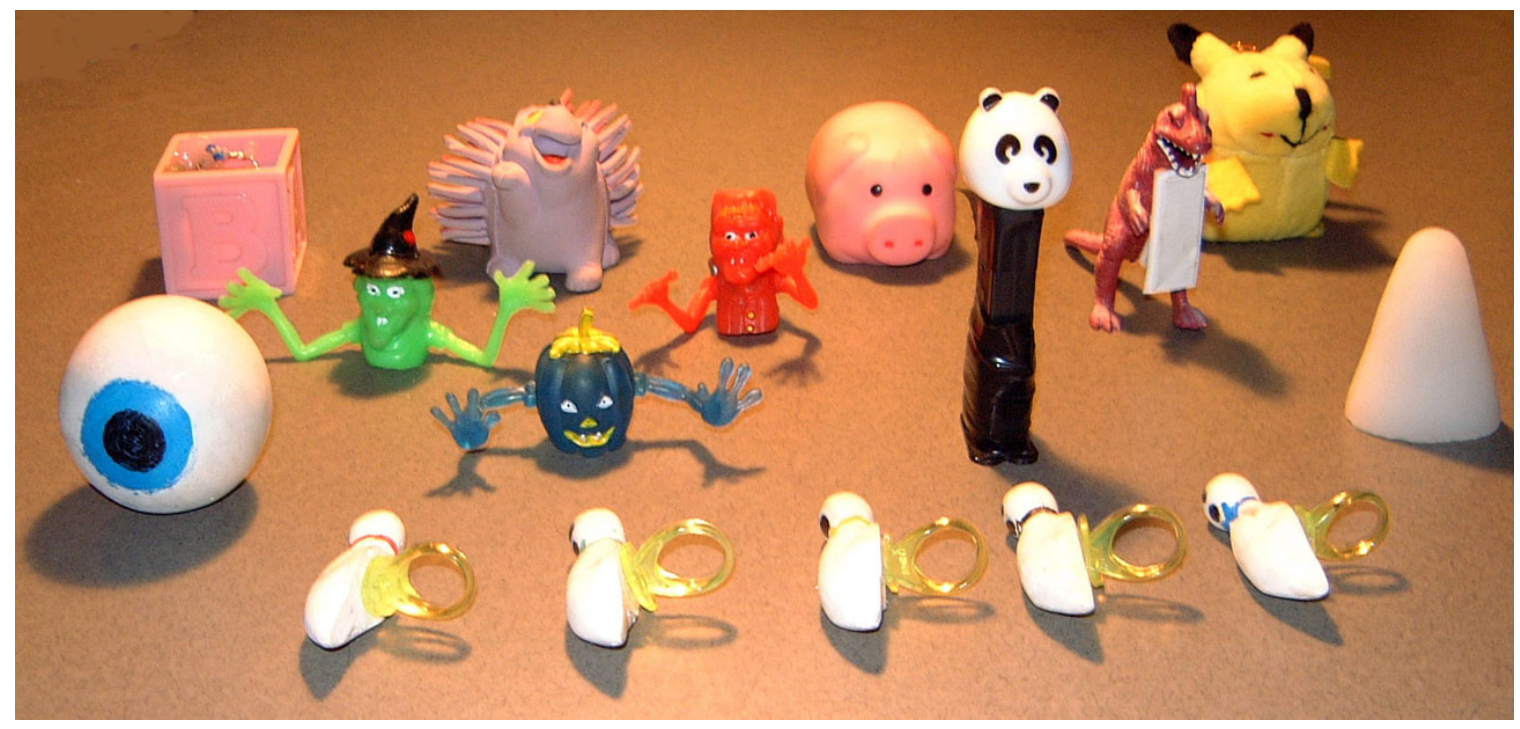

Figure 3: Ensemble of tagged objects that control Musical Trinkets 


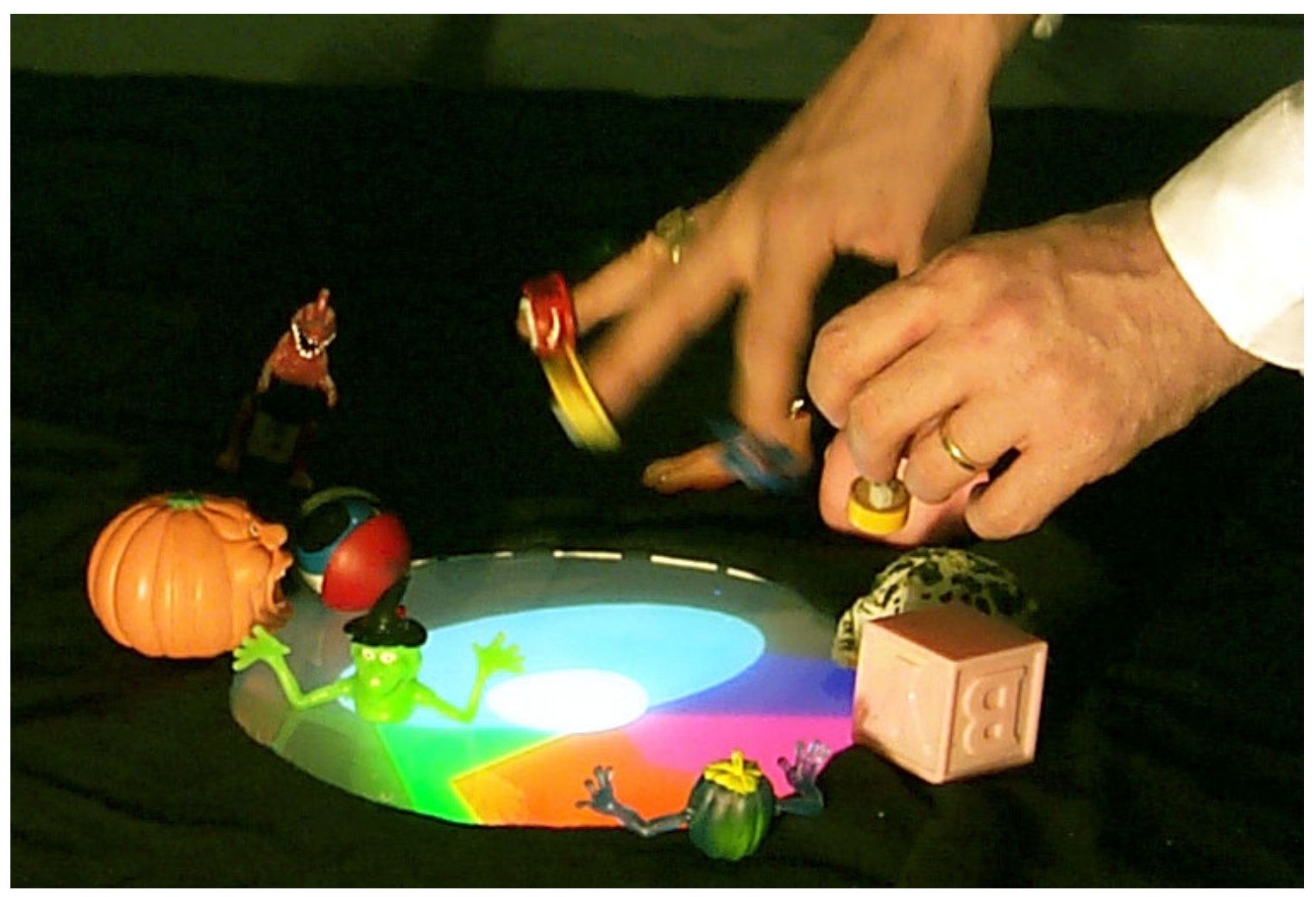

Figure 4: Musical Trinkets running with interactive graphics 


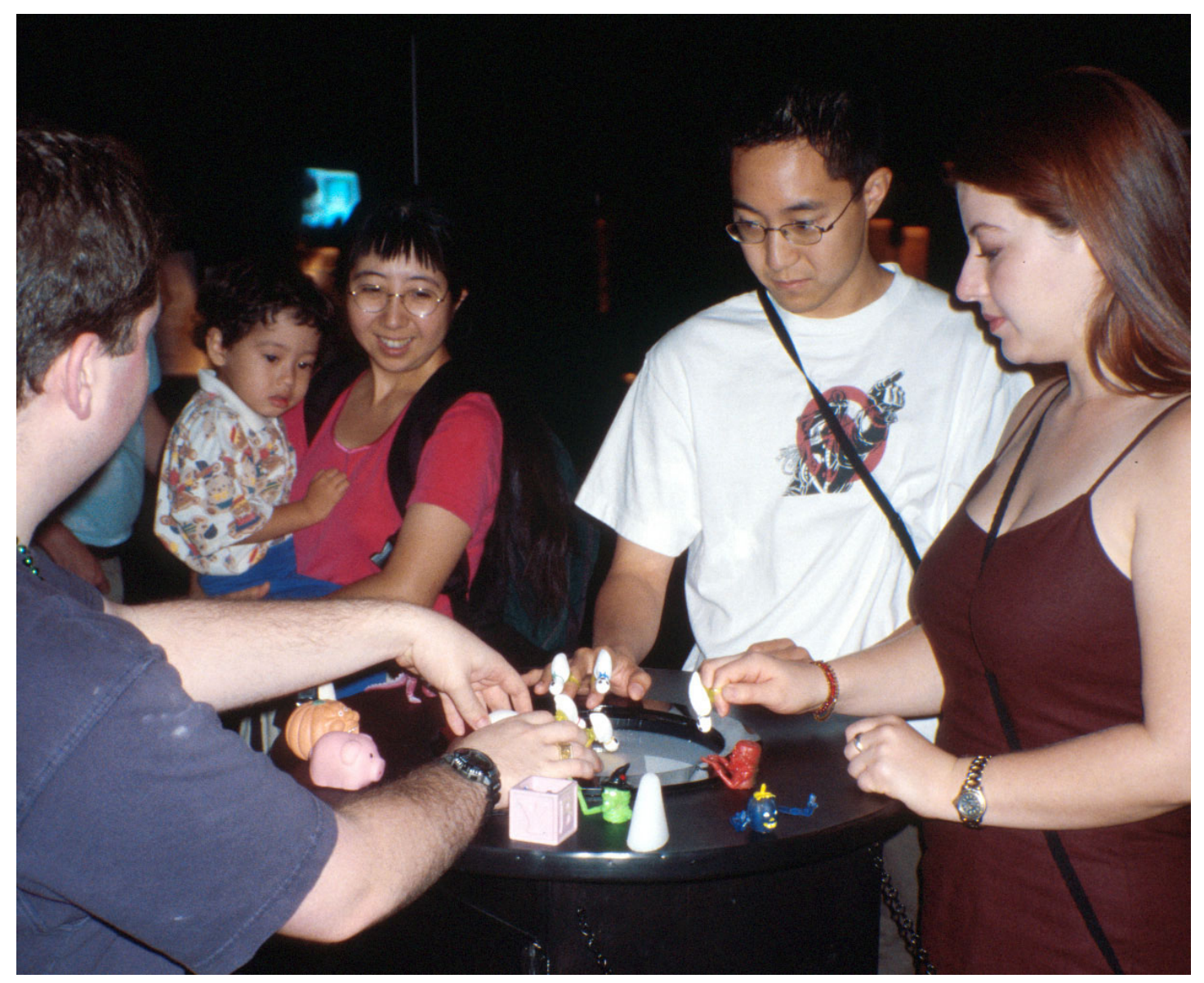

Figure 5: Musical Trinkets at SIGGRAPH 2000 

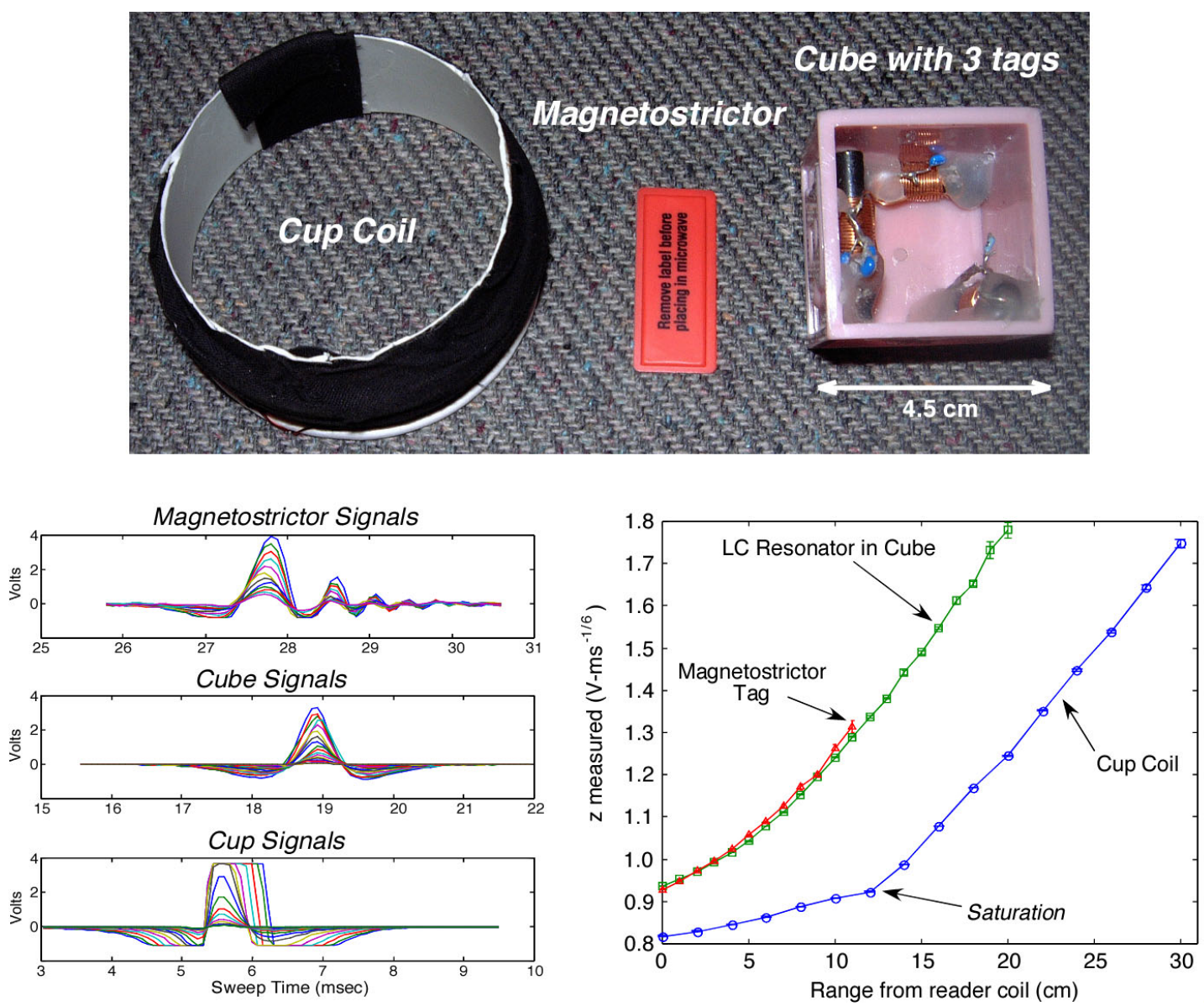

Figure 6: Range response for three different types of tags
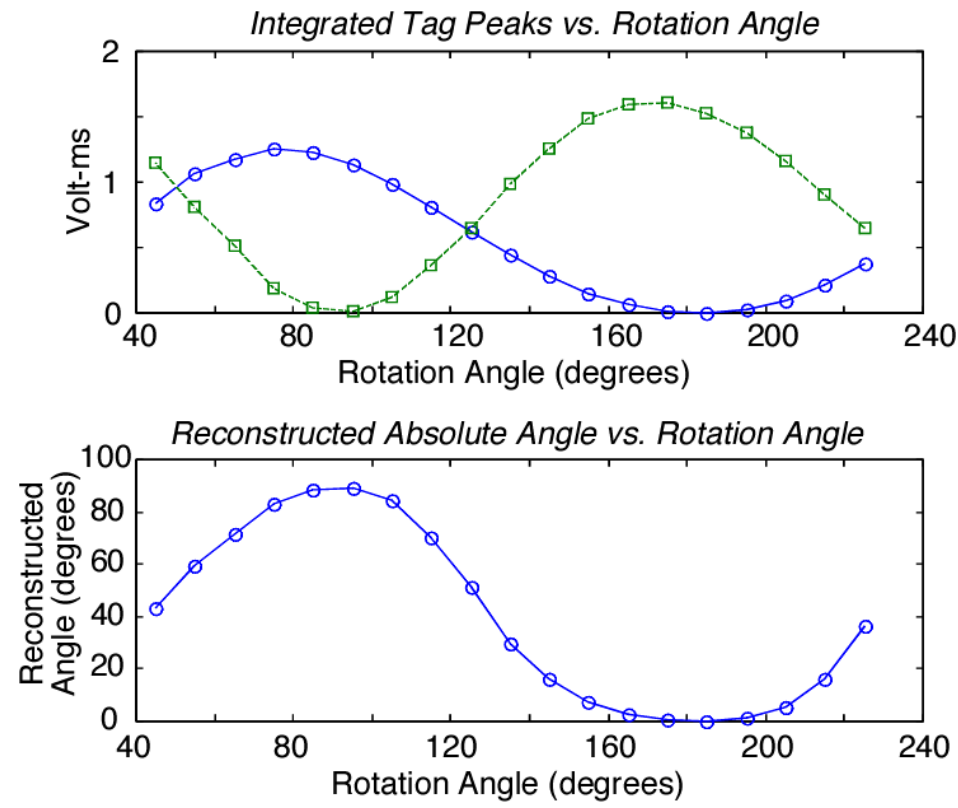

Figure 7: Response of two orthogonal tags in the Cube to rotation at fixed range 


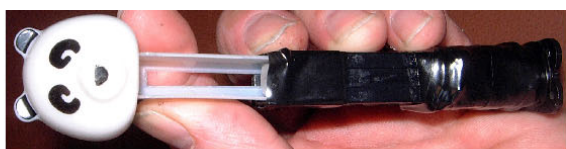

Pez Controller Extended

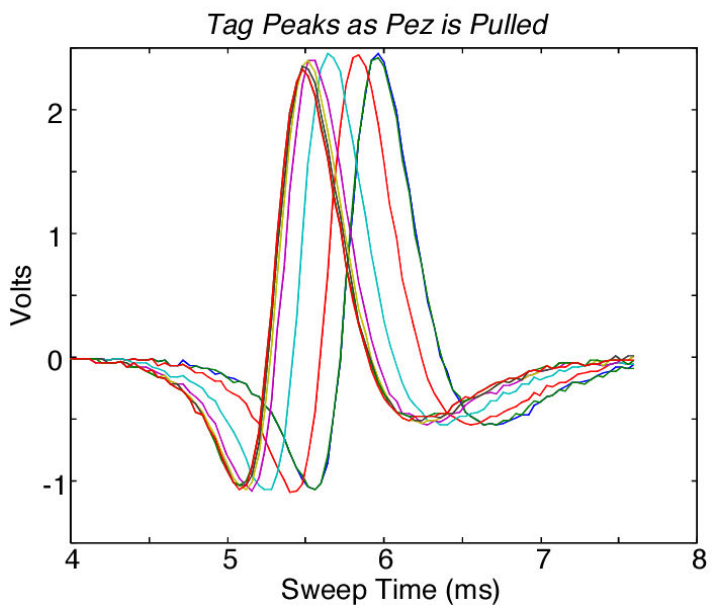

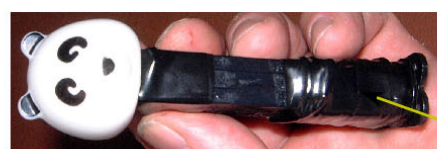

Pez Controller Contracted

Coil wound around outside of Pez's body

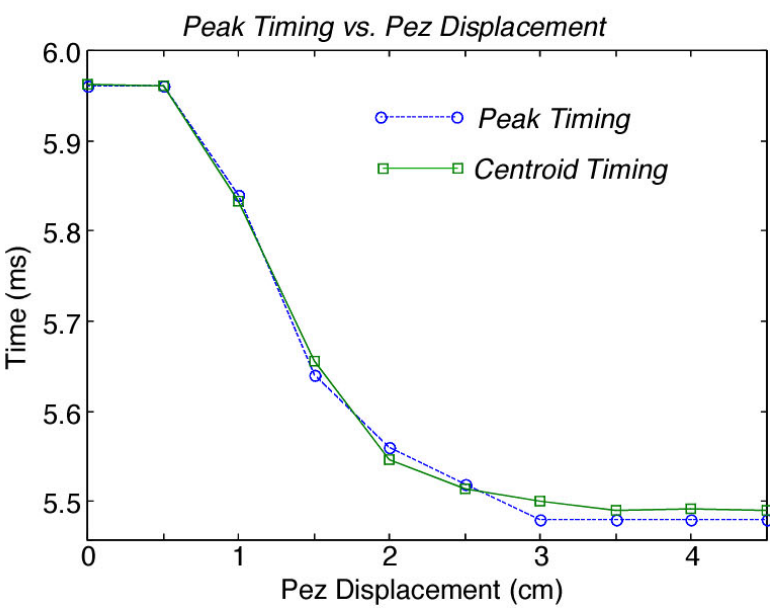

Figure 8: A wireless pull sensor - response of the Pez tag to head displacement

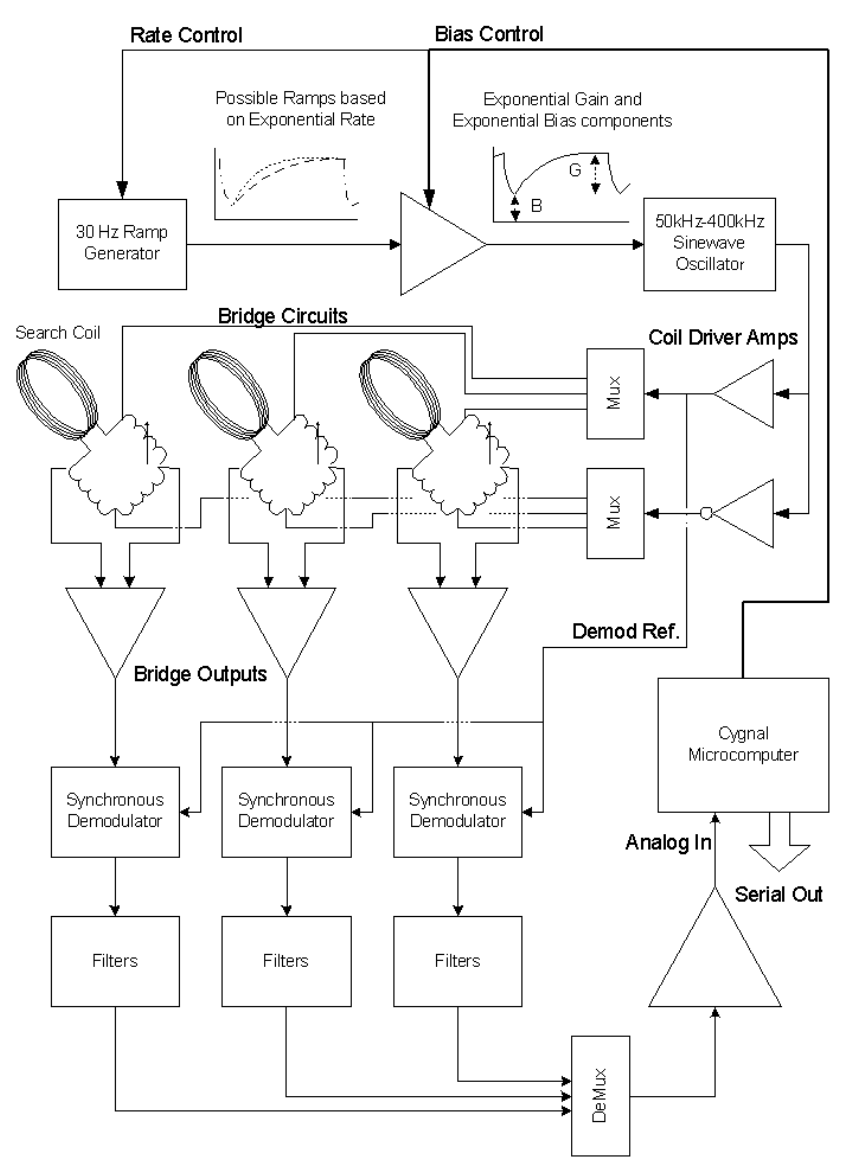

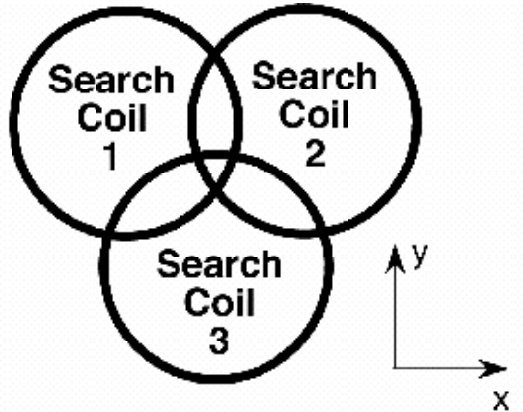

Overhead View

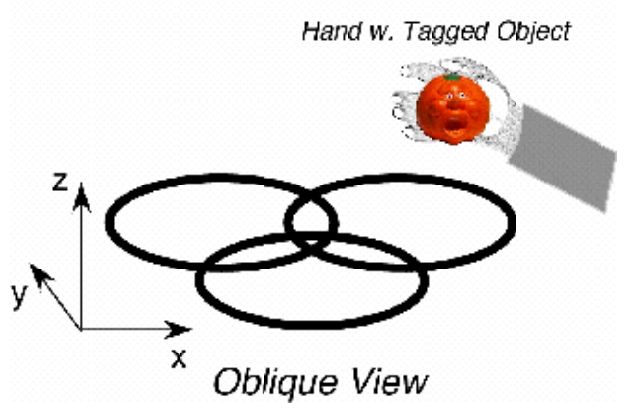

Figure 9: Block Diagram for the Musical Navigatrics tag reader and read coil geometry 


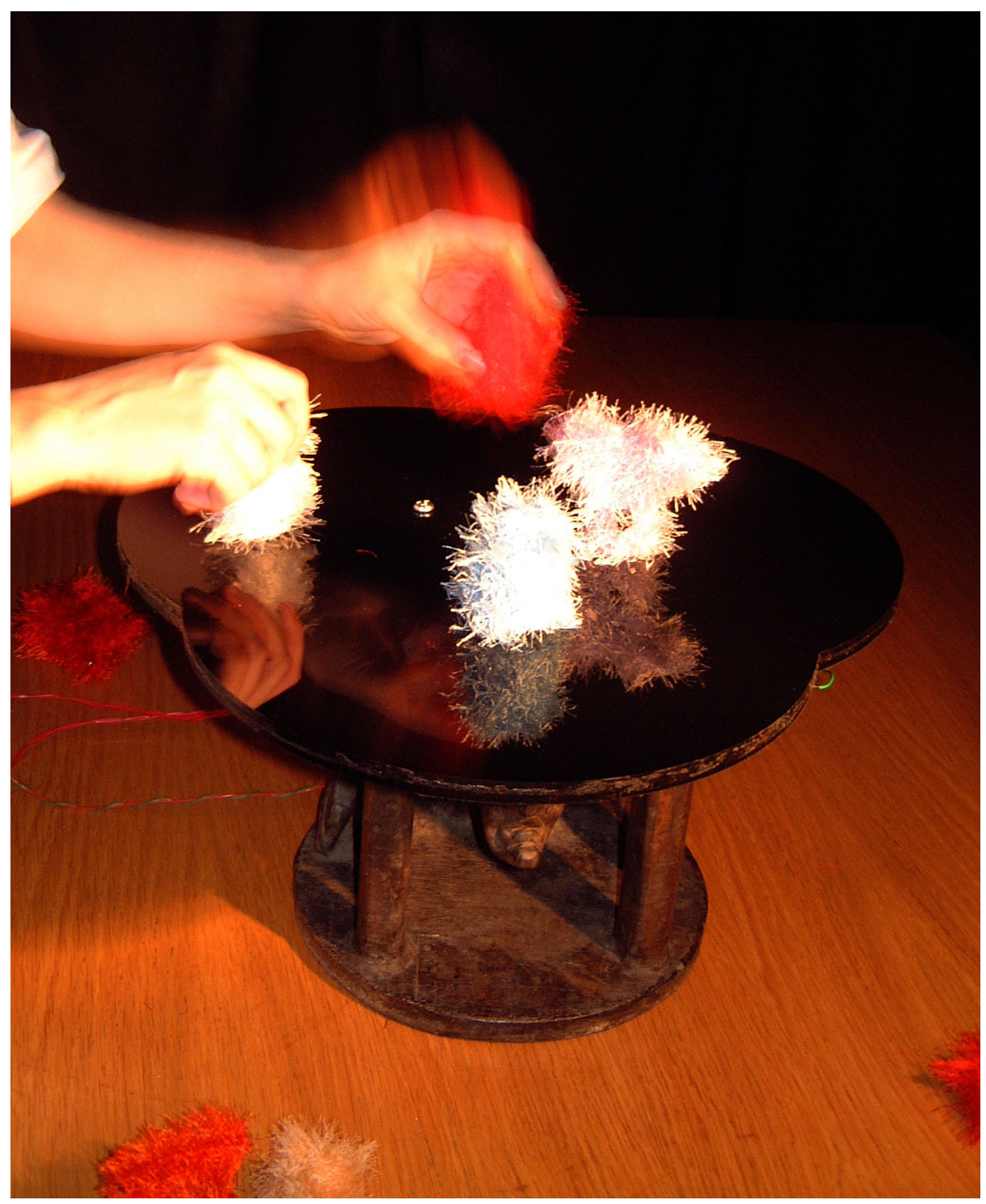

Figure 10: Playing Musical Navigatrics at NIME2002 in Dublin 
Active Tag Module

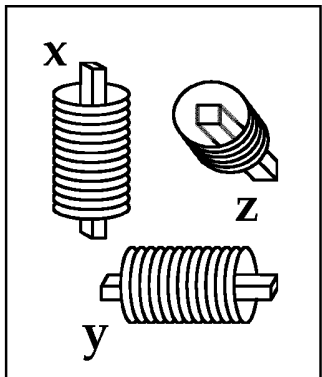

3 orthogonal pickup coils, analog electronics, $\mu$-processor,

communication, power

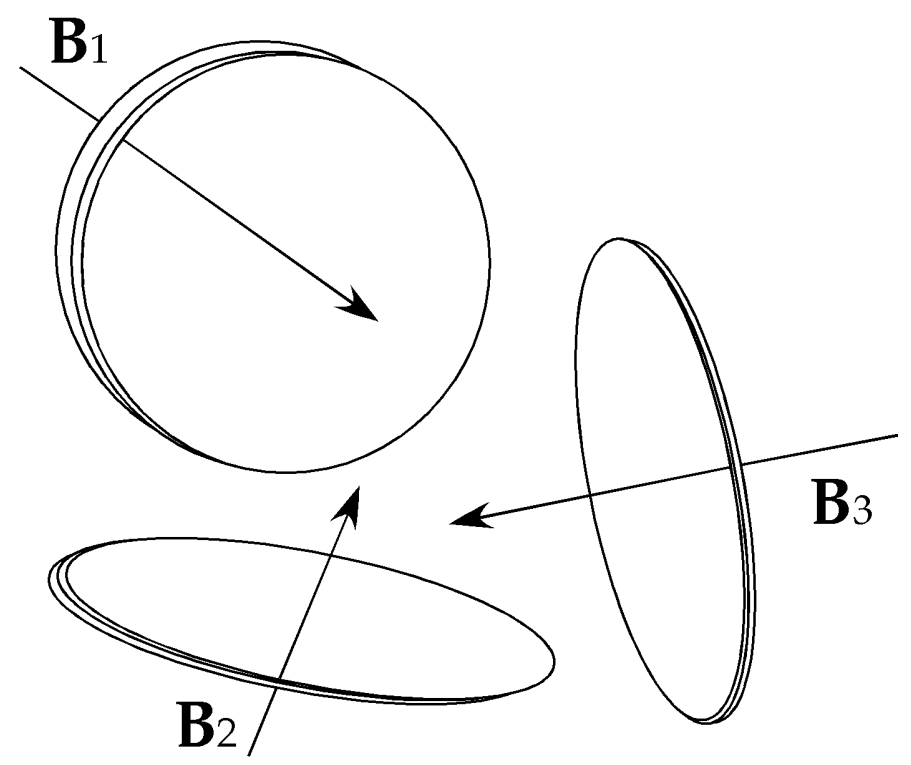

Transmit coils and tangent fields

Figure 11: Coil schematic for active tagging scheme. The large orthogonal reference coils $\left(B_{1}, B_{2}, B_{3}\right)$ successively induce signals into the small orthogonal reference coils $(x, y, z)$, which are actively amplified, detected, and digitized.

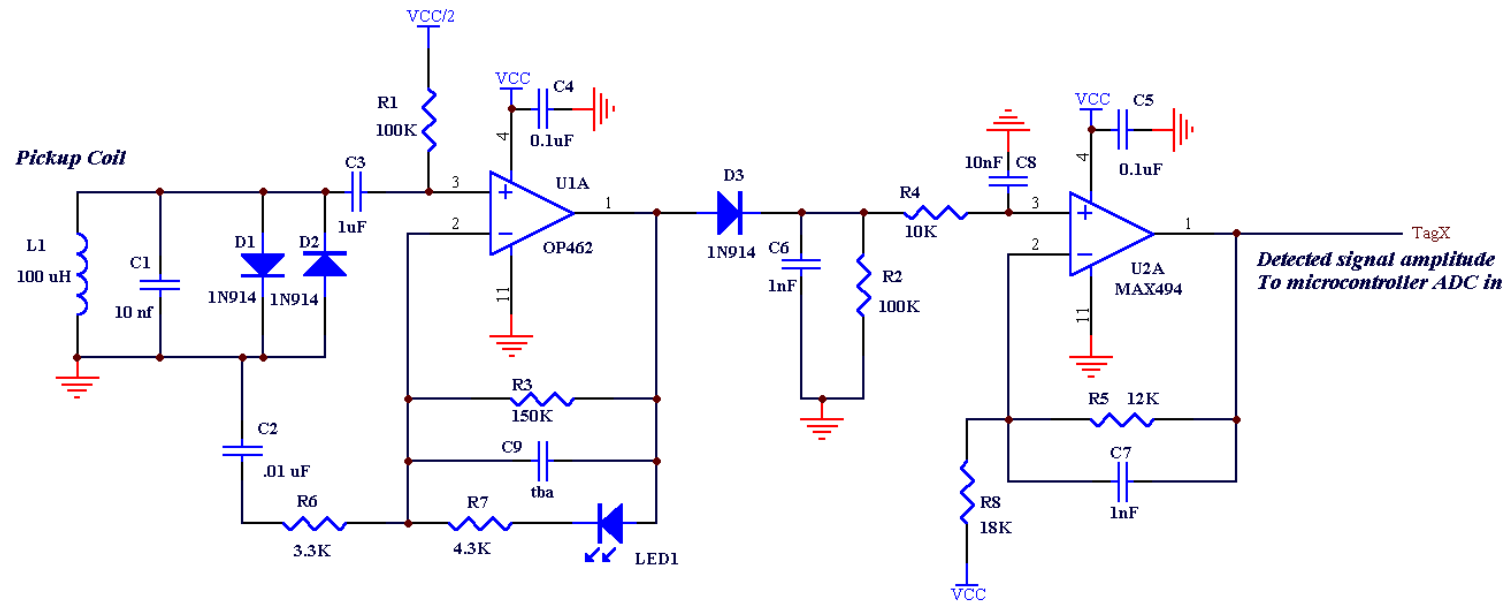

Figure 12: Simple active read electronics conditioning each pickup coil 


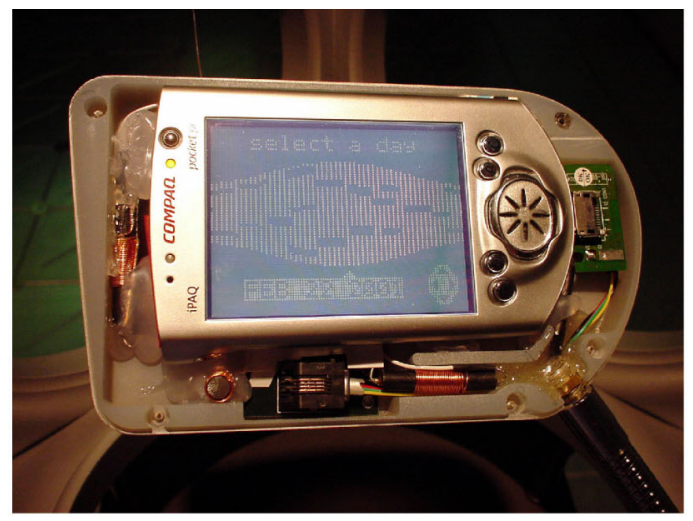

PDA inserted, housing removed
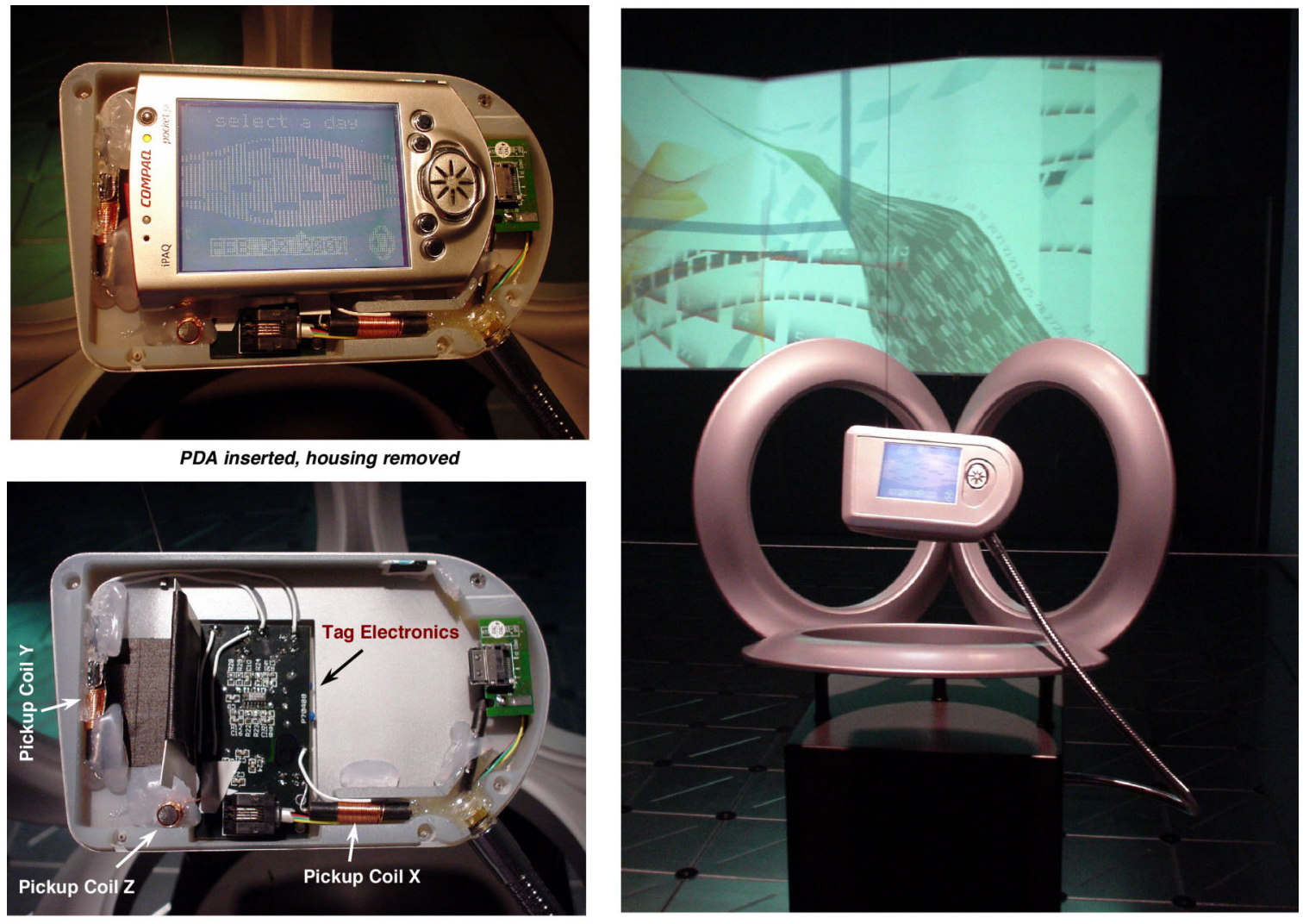

PDA and housing removed

One of three interaction stations showing reference coils and tethered PDA

Figure 13: Active magnetic tracker motoring PDA location in Atmosphere at MOMA 